\title{
Association between Markers of Glycemic Control, Cardiovascular Complications and Survival in Type 2 Diabetic Patients with End-Stage Renal Disease
}

\author{
Tomonari Okada, Toshiyuki Nakao, Hiroshi Matsumoto, Tamami Shino, \\ Yume Nagaoka, Ryo Tomaru and Toshikazu Wada
}

\begin{abstract}
Background and Objective The influence of glycemic control on cardiovascular (CV) complications or survival is not clear in diabetic patients with end-stage renal disease (ESRD). Although glycohemoglobin (HbAlc) is widely used as a marker of hyperglycemia in these patients, it may be unreliable because of shortened erythrocyte lifespan. Glycated albumin (GA) is an alternative marker. We investigated the relation between these markers and development of CV complications or survival in diabetic ESRD patients.

Patients and Methods We obtained three variables as markers of glycemic control: 1) mean HbA1c levels during 1-year after initiation of dialysis (HbAlc1), 2) mean $\mathrm{HbA1c}$ levels during 3 months from August to October 2002 (HbA1c2), 3) GA on October 2002 (GA2) from 78 type 2 diabetic patients on chronic hemodialysis. We examined the influence of these variables on survival or development of $\mathrm{CV}$ diseases using the multivariate Cox proportional-hazards models until September 2006.

Results The 3-year survival rate was 73\%. A total of 27 patients died, 15 from CV diseases. A total of 23 $\mathrm{CV}$ diseases developed in 20 patients. Neither HbA1c1nor HbA1c2 was associated with all-cause mortality, $\mathrm{CV}$ mortality or development of CV diseases. GA2 was also not associated with mortality. However, the higher $\mathrm{GA} 2$ group $(\mathrm{GA} \geqq 23.0 \%$ ) had a significantly higher rate of development of $\mathrm{CV}$ diseases than the lower GA2 group $(\mathrm{GA}<23.0 \%)$ (log-rank test $\mathrm{p}=0.03)$. The higher $\mathrm{GA} 2$ group was significantly associated with development of $\mathrm{CV}$ diseases relative to the lower GA2 group (hazard ratio 3.25, $\mathrm{p}=0.04$ ).

Conclusion Neither HbA1c levels nor GA levels, at initiation of dialysis or on chronic dialysis, predicted mortality in diabetic ESRD patients. However, poor glycemic control as reflected by higher GA levels may be associated with the development of $\mathrm{CV}$ diseases. More studies are needed to clarify the beneficial effect of glycemic control in these patients.
\end{abstract}

Key words: glycemic control, survival, type 2 diabetes, end-stage renal disease, glycohemoglobin, glycated albumin

(DOI: 10.2169/internalmedicine.46.6355)

\section{Introduction}

In diabetic patients, glycemic control has an essential role in preventing the development and progression of microvascular or cardiovascular (CV) complications (1-4). Glycohemoglobin (HbA1c) is the most representative marker of hyperglycemia for assessing glycemic control. In diabetic patients with end-stage renal disease (ESRD), there are two unresolved and important problems. First, it is not clear whether glycemic control reduces the risk of cardiovascular complications or mortality. Second, it is not clear whether $\mathrm{HbAlc}$ is a reliable marker of hyperglycemia (5).

Only a few studies have shown beneficial impact of predialysis glycemic control on survival after initiation of dialysis $(6,7)$. In addition, limited studies showed an association of poor glycemic control with poor survival on chronic dialysis $(8,9)$. However, several studies showed no correlation 
Table 1. Patient Characteristics

\begin{tabular}{|c|c|}
\hline Age (years) (range) & $63 \pm 10(33-86)$ \\
\hline Male/ Female (n) & $60 / 18$ \\
\hline Duration of dialysis (months) & $49 \pm 32(12-192)$ \\
\hline Body weight (kg) & $59.3 \pm 10.2(39.0-94.5)$ \\
\hline Body mass index $\left(\mathrm{kg} / \mathrm{m}^{2}\right)$ & $22.5 \pm 3.2(16.6-32.5)$ \\
\hline Systolic blood pressure $(\mathrm{mmHg})$ & $161 \pm 19(119-217)$ \\
\hline Diastolic blood pressure $(\mathrm{mmHg})$ & $79 \pm 13(36-107)$ \\
\hline Intradialytic weight gain (\%) & $4.5 \pm 2.0(-0.6-8.5)$ \\
\hline Hematocrit (\%) & $32.4 \pm 2.5(25.9-40.1)$ \\
\hline Serum albumin (g/dl) & $3.83 \pm 0.26(3.10-4.37)$ \\
\hline Serum phosphorus (mg/dl) & $5.75 \pm 1.11(3.13-8.90)$ \\
\hline Blood glucose (mg/dl) & $163 \pm 43(87-260)$ \\
\hline Glycohemoglobin (\%) & $6.28 \pm 1.05(4.17-8.67)$ \\
\hline Glycated albumin (\%) & $23.6 \pm 5.1(15.4-38.6)$ \\
\hline $\mathrm{KT} / \mathrm{V}$ & $1.17 \pm 0.16(0.78-1.61)$ \\
\hline Therapy for diabetes $(n)$ & Diet 33 / oral hypoglycemic agents 13 / Insulin 32 \\
\hline \multirow[t]{2}{*}{ Erythropoietin doses (U/kg/week) (n) } & $4150 \pm 3480(0-13500)$ \\
\hline & $\begin{array}{l}\text { No use, } 17 / \leqq 3000 \mathrm{U} / \text { week, } 25 / 3000 \text { to } 6000 \mathrm{U} / \text { week, } 14 \text { I } \\
6000 \text { to } 9000 \mathrm{U} / \text { week , } 20 />9000 \mathrm{U} / \text { week; } 2\end{array}$ \\
\hline History of CV diseases (n) & $\begin{array}{l}\text { Ischemic heart disease 12/ Cerebrovascular disease 5/ } \\
\text { Peripheral vascular disease } 3\end{array}$ \\
\hline
\end{tabular}

between glycemic control and survival $(10,11)$. Therefore, the significance of glycemic control is not established and appropriate targets of glycemic control have not yet been determined in diabetic ESRD patients.

HbA1c level is influenced by erythrocyte lifespan or erythropoietin therapy $(12,13)$. Shortened erythrocyte lifespan or erythropoietin therapy changes HbA1c levels independent of glycemic control. It is generally known that HbA1c levels tend to be lower in ESRD patients. Glycated albumin (GA) is an alternative marker of glycemic control and is not influenced by erythrocyte lifespan or erythropoietin therapy (14). GA levels may reflect blood glucose levels more correctly in ESRD patients (15). There have been no previous studies concerning the relation between GA levels and the prognosis in these patients.

To clarify the association between glycemic control and clinical outcome in diabetic ESRD patients, we obtained both HbA1c and GA levels simultaneously on chronic dialysis and examined the association with prognosis. In addition, we also obtained HbA1c levels at initiation of dialysis and examined the association with prognosis.

\section{Subjects and Methods}

A total of 78 type 2 diabetic ESRD patients receiving chronic hemodialysis in 4 outpatient dialysis centers in Tokyo were entered in the present study. Patients who had received hemodialysis for at least 12 months by October 2002 and who had no liver cirrhosis or unhealed malignancy, were selected. The diagnosis of diabetic nephropathy as the cause of renal disease was based on duration of diabetes, existence of diabetic retinopathy or other clinical data. We obtained HbA1c values at initiation of dialysis, after 6 months, and after 12 months. In addition, we obtained HbA1c values on 3 occasions from August to October 2002 and a single GA value on October 2002. After that, we followed patients until September 2006. The duration of dialysis until October 2002 was $48 \pm 32$ (12-128) months. The mean follow-up period from October 2002 was $35 \pm 16$ (248) months. Clinical characteristics of patients are shown in Table 1. Clinical and laboratory data were collected on 3 occasions from August to October 2002 and the 3-month average values were used for each patient. Blood pressure was measured in the supine position before the regular hemodialysis session. Blood samples for laboratory data were obtained at the start of the hemodialysis session.

Non-fasting blood glucose was also obtained at the start of the hemodialysis session on 3 occasions during 3 months and the average values were used for each patient. Blood glucose was measured about 2 hours after breakfast in 66 patients receiving the morning hemodialysis session. It was measured about 4 hours after lunch in 12 patients receiving the evening hemodialysis session.

We adopted three variables as markers of glycemic control: 1) mean $\mathrm{HbA} 1 \mathrm{c}$ levels during 1 year after initiation of dialysis $(H b A 1 c 1)(n=69)$, those were the mean value at initiation of dialysis, after 6 months and after 12 months, 2) mean HbA1c levels during 3 months from August to October 2002 (HbA1c2), and GA on October 2002 (GA2). We examined the influence of these variables on all-cause mortality, $\mathrm{CV}$ mortality, or development of $\mathrm{CV}$ diseases using the multivariate Cox proportional-hazards models. The co- 
Table 2. Newly Diagnosed Cardiovascular Diseases and Causes of Death

\begin{tabular}{|c|c|c|}
\hline \multicolumn{3}{|l|}{ Causes of death $(n)$} \\
\hline \multirow[t]{4}{*}{ Cardiovascular diseases } & Myocardial infarction & 5 \\
\hline & Sudden death & 5 \\
\hline & Cerebral hemorrhage & 4 \\
\hline & Congestive heart failure & 1 \\
\hline \multirow[t]{5}{*}{ Non-cardiovascular diseases } & Infectious diseases & 3 \\
\hline & Respiratory failure & 3 \\
\hline & Malignancy & 2 \\
\hline & Traffic accident & 1 \\
\hline & Unknown causes & 3 \\
\hline \multirow[t]{6}{*}{ Development of cardiovascular diseases ( $n$ ) } & Myocardial infarction & 2 \\
\hline & Ischemic heart disease & 4 \\
\hline & Cerebral infarction & 8 \\
\hline & Cerebral hemorrhage & 3 \\
\hline & Peripheral vascular disease & 5 \\
\hline & Complete AV block & 1 \\
\hline
\end{tabular}

variates included age, gender, duration of dialysis, body mass index, systolic blood pressure, intradialytic weight gain, hematocrit, serum albumin, serum phosphorus, KT/V and history of $\mathrm{CV}$ diseases in multivariate analysis. Hazard ratios (HR) were presented per unit $(1 \%)$ increase in HbAlc or GA and $95 \%$ confidence intervals (CI) were calculated for the HR. Furthermore, we divided subjects into three sets of two groups according to the median values of HbA1c1, HbA1c2 and GA2, respectively. We examined the KaplanMeier survival curves of each set of two groups for each marker. We also examined HR of the group of the higher values relative to the lower values for each marker.

Development of CV diseases included ischemic heart disease, arrhythmia requiring treatment by permanent pacemaker, cerebral infarction, cerebral hemorrhage, and peripheral vascular disease. Ischemic heart disease included definite coronary artery disease diagnosed by coronary angiography, myocardial infarction or treatment by percutaneous coronary intervention or coronary artery bypass graft. Peripheral vascular disease included treatment by percutaneous transluminal angioplasty or other vascular surgery. Causes of death related to $\mathrm{CV}$ complications included sudden death, heart failure, myocardial infarction, cerebral infarction, and cerebral hemorrhage. Informed consent to use clinical data in this study was obtained from all patients.

Both HbA1c was measured by latex agglutination immunoassay (RAPIDIA AUTO; SRL, Tokyo, Japan) and the normal range was $4.3-5.8 \%$. GA was measured by enzymatic method using albumin-specific proteinase, ketoamine oxidase and albumin assay reagent (Lucica GA; Asahi Kasei Pharma Co., Tokyo, Japan) and the normal range was 12.4-
$16.3 \%$ (16).

Changes in the two groups were examined by Wilcoxon's signed-rank test. Correlation between two values was analyzed by Pearson's correlation coefficient. All values were expressed as means $\pm \mathrm{SD}$, and a $\mathrm{P}$ value of less than 0.05 was considered to indicate a statistically significant difference. Statistical analysis was performed with a StatView computer program (Abacus Concepts, Berkely, CA, USA).

\section{Results}

\section{Survival and development of CV complications}

The 1-, 3- and 4-year survival rates during the follow-up period were $90.8 \%, 73.1 \%$ and $60 \%$, respectively. During follow-up, 27 patients died, 15 from $\mathrm{CV}$ diseases. A total of 23 cases of $\mathrm{CV}$ diseases developed in 20 patients. Causes of death and newly diagnosed $\mathrm{CV}$ diseases are shown in Table 2 .

\section{Glycemic control status and relationship between markers of glycemic control}

Mean values (median values) of HbA1c1, HbA1c2 and GA2 were $6.53 \pm 1.16 \%(6.43 \%), 6.28 \pm 1.05 \%$ (6.20\%) and $23.6 \pm 5.1 \%(23.0 \%)$, respectively. Eight (11.6\%) patients had HbA1c1 greater than $8.0 \%$ and $5(6.4 \%)$ patients had HbA1c2 greater than $8.0 \%$.

Relationship between HbA1c2 and GA2 levels are shown in Fig. 1. The correlation coefficients between HbA1c2 and GA2 levels, those between HbA1c2 and blood glucose levels, and those between GA2 and blood glucose levels were 
$0.53,0.42$ and 0.41 , respectively (all $\mathrm{p}<0.001$ ).

Influence of markers of glycemic control on survival or development of $\mathrm{CV}$ complications using multivariate Cox proportional-hazards models and Kaplan-Meier survival curves

A $1 \%$ increase in HbA1c1, HbA1c2 or GA2 was not significantly associated with all-cause mortality, CV mortality or development of CV diseases in Cox models (Table 3).

In the model including GA2, a history of CV diseases remained a significant predictor of all-cause mortality (HR, 3.91; $95 \% \mathrm{CI}, 1.54$ to $9.90 ; \mathrm{p}=0.004$ ), CV mortality (HR, 3.81; $95 \% \mathrm{CI}, 1.07$ to $13.51 ; \mathrm{p}=0.04$ ) or development of $\mathrm{CV}$ diseases (HR, 4.83 ; 95\% CI, 1.26 to $18.60 ; \mathrm{p}=0.02$ ) and systolic blood pressure remained a significant predictor of de-

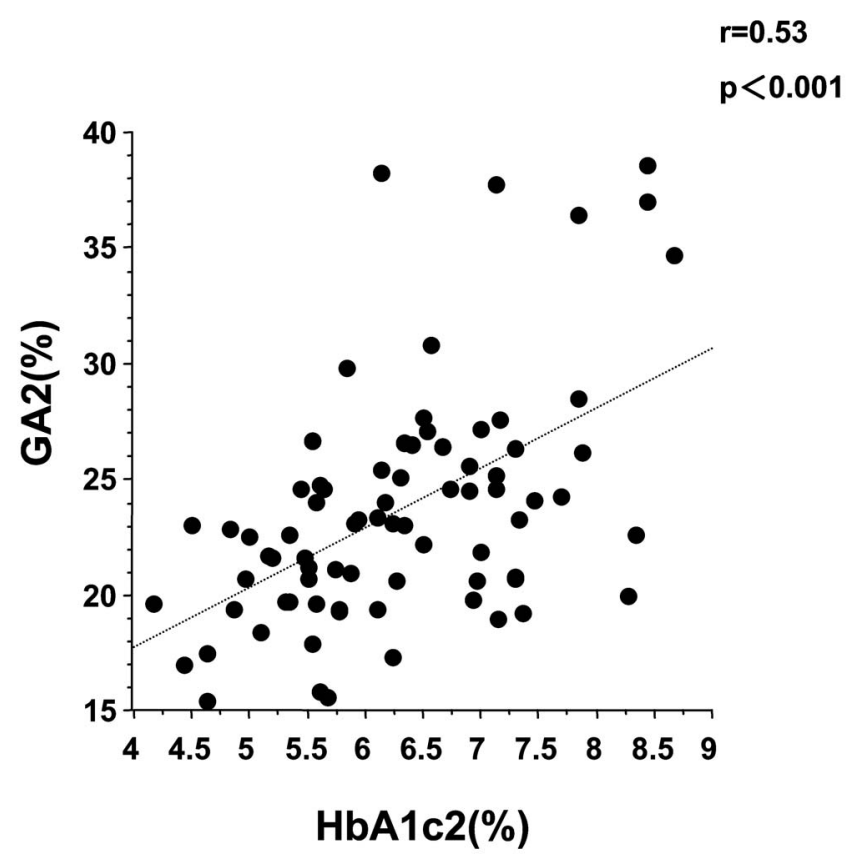

Figure 1. Correlation between HbA1c2 and GA2. velopment of $\mathrm{CV}$ diseases (HR, 1.03; 95\% CI, 1.00 to 1.07; $\mathrm{p}=0.04)$.

In the model including $\mathrm{HbA} 1 \mathrm{c} 2$, a history of $\mathrm{CV}$ diseases remained a significant predictor of all-cause mortality (HR, 3.89; $95 \% \mathrm{CI}, 1.53$ to $9.88 ; \mathrm{p}=0.004$ ) or development of $\mathrm{CV}$ diseases (HR, 5.35; 95\% CI, 1.37 to 20.94; $\mathrm{p}=0.02$ ) and systolic blood pressure remained a significant predictor of development of CV diseases (HR, 1.04; 95\% CI, 1.01 to 1.07; $\mathrm{p}=0.02$ ).

In the model including $\mathrm{HbA1c} 1$, a history of $\mathrm{CV}$ diseases remained a significant predictor of all-cause mortality (HR, 4.94; 95\%CI, 1.68 to $14.52 ; \mathrm{p}=0.004$ ) or CV mortality (HR, 9.71; $95 \% \mathrm{CI}, 1.49$ to $63.11 ; \mathrm{p}=0.02$ ).

\section{The Kaplan-Meier survival curves and Cox models using the groups according to the median values of each glycemic marker}

The Kaplan-Meier survival curves showed no significant differences in mortality or development of $\mathrm{CV}$ diseases between the higher $\mathrm{HbA} 1 \mathrm{c} 2(\mathrm{HbA} 1 \mathrm{c} \geqq 6.20 \%)$ group and the lower HbA1c2 (HbA1c < 6.20\%) group (Fig. 2). The cases of $\mathrm{HbAlc1}$ were the same. There were also no significant differences in mortality between the higher GA2 (GA $\geqq$ $23.0 \%)$ group and the lower GA2 $(\mathrm{GA}<23.0 \%)$ group (Figs. 3a, 3b). On the other hand, CV diseases developed in 15 patients in the higher GA2 group $(\mathrm{n}=39)$ and in $5 \mathrm{pa}-$ tients in the lower GA2 group $(n=39)$. The higher GA2 group had a significant higher rate of development of CV diseases than the lower GA2 group (Fig. 3c) (log-rank test $\mathrm{p}=0.03$ ).

$\mathrm{HbA1c1}$ or HbA1c2 groups were not significantly associated with mortality or development of CV diseases according to the Cox models (Table 4). GA2 groups were also not significantly associated with mortality (Table 4). On the other hand, the higher GA2 group was significantly associated with development of $\mathrm{CV}$ diseases relative to the lower GA2 group (HR, 3.25; 95\% CI, 1.04 to 10.19; $\mathrm{p}=0.04$ ).

Table 3. Relationship of Glycemic Markers with All-cause Mortality, CV Mortality and Development of CV Diseases

\begin{tabular}{lcccccc}
\hline & All-cause mortality & CV mortality & & $\begin{array}{c}\text { Development of } \\
\text { CV diseases }\end{array}$ \\
& HR $(95 \% \mathrm{Cl})$ & $\mathrm{p}$ & $\mathrm{HR}(95 \% \mathrm{Cl})$ & $\mathrm{p}$ & $\mathrm{HR}(95 \% \mathrm{Cl})$ & $\mathrm{p}$ \\
\hline HbA1c1 (per 1\%) & $1.11(0.71$ to 1.74$)$ & 0.66 & $1.04(0.48$ to 2.28$)$ & 0.92 & $1.34(0.78$ to 2.29$)$ & 0.29 \\
HbA1c2 (per 1\%) & $0.99(0.63$ to 1.56) & 0.98 & $0.78(0.41$ to 1.48$)$ & 0.45 & $1.38(0.76$ to 2.50$)$ & 0.29 \\
GA2 (per 1\%) & $0.98(089$ to 1.08) & 0.69 & $0.88(0.75$ to 1.03$)$ & 0.12 & $1.09(0.96$ to 1.24$)$ & 0.17 \\
\hline
\end{tabular}

$\mathrm{HR}$, hazard ratio; $\mathrm{Cl}$, confidence interval; $\mathrm{CV}$, cardiovascular

Adjusted for age, gender, duration of dialysis, body mass index, systolic blood pressure, intradialytic weight gain, hematocrit, serum albumin, serum phosphorus, KT/V and history of $\mathrm{CV}$ diseases 


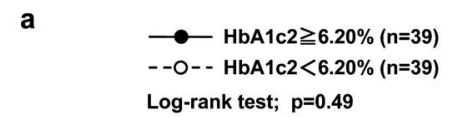

\section{b}

Log-rank test; $p=0.49$
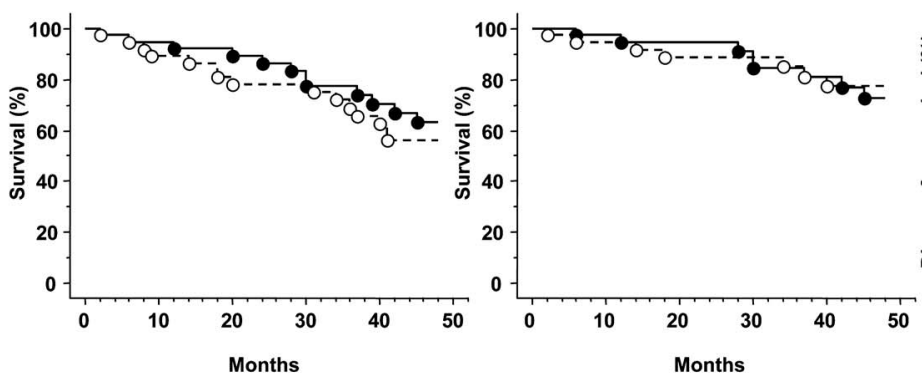

c

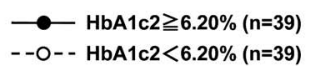
- -O- - HbA1c2 $<6.20 \%$ ( $n=39)$ Log-rank test; $p=0.85$ Log-rank test; $p=0.58$

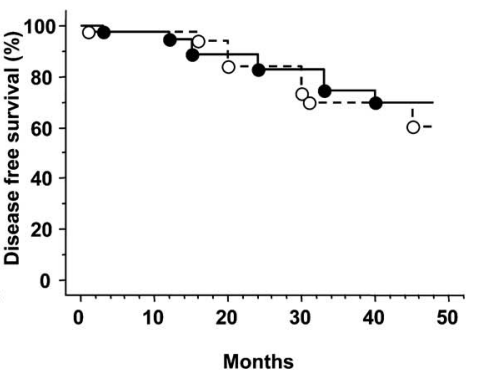

Figure 2. The Kaplan-Meier survival curves of all-cause mortality (a), CV mortality (b) and development of $\mathrm{CV}$ diseases (c) in the higher HbA1c2 (HbA1c $\geqq 6.20 \%$ ) group and the lower HbA1c2 (HbA1c $<6.20 \%)$ group.

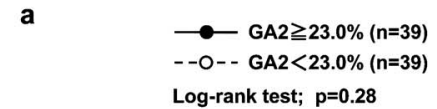

b Log-rank test; $p=0.28$

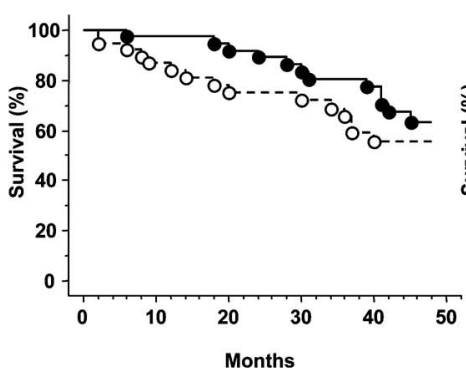

$\longrightarrow-$ GA2 $\geqq 23.0 \%(n=39)$ - -O- - GA2 $<23.0 \%(n=39)$ Log-rank test; $p=0.10$

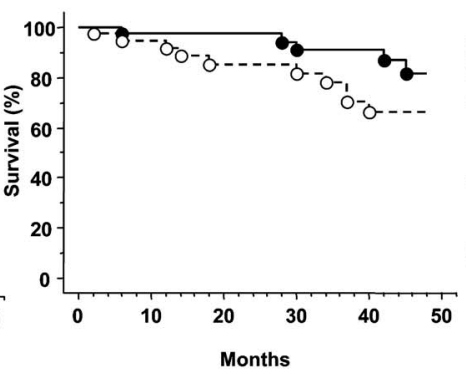

c

$\longrightarrow-\mathrm{GA} 2 \geqq 23.0 \%(n=39)$ - -O- - GA2<23.0\% (n=39) Log-rank test; $p=0.03$

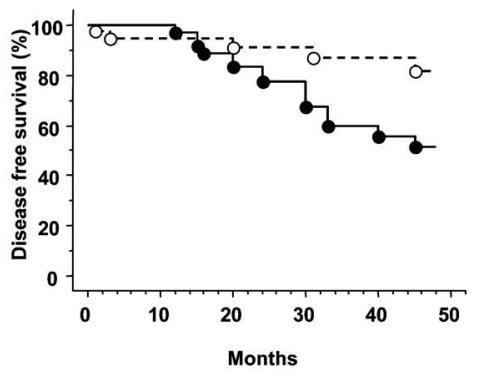

Figure 3. The Kaplan-Meier survival curves of all-cause mortality (a), CV mortality (b) and development of CV diseases (c) in the higher GA2 (GA $\geqq 23.0 \%)$ group and the lower GA2 (GA < 23.0\%) group.

Table 4. Relationship of the Groups According to Higher or Lower Values of Glycemic Markers with All-cause Mortality, CV Mortality and Development of CV Diseases

\begin{tabular}{|c|c|c|c|c|c|c|}
\hline & All-cause mortality & & CV mortality & & $\begin{array}{l}\text { Development of } \\
\text { CV diseases }\end{array}$ & \\
\hline & $\mathrm{HR}(95 \% \mathrm{Cl})$ & p & $\mathrm{HR}(95 \% \mathrm{Cl})$ & $\mathbf{p}$ & HR (95\%Cl) & $\mathbf{p}$ \\
\hline \multicolumn{7}{|l|}{ HbA1c1 } \\
\hline lower group $(<6.43 \%)$ & 1 & & 1 & & 1 & \\
\hline higher group ( $\geqq 6.43 \%$ ) & $0.93(0.34$ to 2.58$)$ & 0.90 & $1.06(0.16$ to 7.12$)$ & 0.95 & $0.81(0.27$ to 2.46$)$ & 0.72 \\
\hline \multicolumn{7}{|l|}{ HbA1c2 } \\
\hline lower group $(<6.20 \%)$ & 1 & & 1 & & 1 & \\
\hline higher group ( $\geqq 6.20 \%$ ) & 1.20 (0.48 to 2.99$)$ & 0.70 & $1.65(0.47$ to 5.76$)$ & 0.44 & $0.92(0.30$ to 2.86$)$ & 0.89 \\
\hline \multicolumn{7}{|l|}{ GA2 } \\
\hline lower group $(<23.0 \%)$ & 1 & & 1 & & 1 & \\
\hline higher group ( $\geqq 23.0 \%$ ) & $0.76(0.31$ to 1.84$)$ & 0.54 & $0.34(0.12$ to 1.46$)$ & 0.17 & 3.25 (1.04 to 10.19$)$ & 0.04 \\
\hline
\end{tabular}

HR, hazard ratio; $\mathrm{Cl}$, confidence interval; CV, cardiovascular

Adjusted for age, gender, duration of dialysis, body mass index, systolic blood pressure, intradialytic weight gain, hematocrit, serum albumin, serum phosphorus, KTN and history of CV diseases

\section{Effect of changes in HbA1c levels on prognosis}

We divided subjects into two groups according to the changes between $\mathrm{HbA} 1 \mathrm{c} 1$ and $\mathrm{HbA} 1 \mathrm{c} 2$ levels. The group with an increase of HbA1c levels (HbA1c2 levels>HbA1c1 levels) consisted of 29 patients and the mean change value was $0.63 \pm 0.58 \%$. The group with a decrease of HbAlc levels (HbA1c1 levels $>\mathrm{HbA} 1 \mathrm{c} 2$ levels) consisted of 40 patients 
and the mean change value was $-0.87 \pm 0.75 \%$. The KaplanMeier survival curves showed no significant differences in mortality or development of $\mathrm{CV}$ diseases between the increased group and the decreased group.

\section{Discussion}

Our results showed no significant associations between markers of glycemic control and survival in type 2 diabetic ESRD patients. The markers both at initiation of dialysis and on chronic dialysis did not predict mortality. A history of CV diseases and systolic blood pressure remained significant predictors of mortality in multivariate analysis.

We suggest several reasons for lack of associations between markers of glycemic control and survival. First, diabetic ESRD patients have already far advanced microvascular and macrovascular complications. Secondly, risk factors other than glycemic control may have more significant effects on $\mathrm{CV}$ complications and survival in these patients.

In the general population or in diabetic patients without renal dysfunction, the association between glucose levels and CV complications or survival was demonstrated in some previous studies (17-19). However, diabetic ESRD patients have already advanced atherosclerosis $(20,21)$. In addition to hyperglycemia, there are many risk factors associated with CV diseases, including traditional factors and uremiarelated factors in diabetic ESRD patients (22). Some previous studies have demonstrated that increased all-cause mortality or CV mortality are associated with advanced atherosclerosis independent of diabetes in ESRD patients (23-25). Therefore, the direct influence of hyperglycemia on fatal CV diseases might be small in these patients.

Non-CV diseases such as infectious diseases or cancer may also influence the outcome in diabetic ESRD patients. Hyperglycemia impairs the host defense and increases the risk of infectious diseases (26). Diabetes increases the risk of cancer in some epidemiological studies (27). However, the influence of glycemic control on the development of infectious diseases or cancer is unclear in diabetic ESRD patients. Our results could not find these associations. The registry data of the Japanese Society for Dialysis Therapy also showed no significant differences of frequency of infectious diseases or cancer as causes of death between diabetic patients and non-diabetic patients (28).

The direct influence of hyperglycemia on the progression of atherosclerosis or development of $\mathrm{CV}$ diseases is not clear in diabetic ESRD patients. Our results showed an association between hyperglycemia, reflected by higher GA levels and development of CV diseases. Several mechanisms of toxic effects of hyperglycemia have been demonstrated, including endothelial dysfunction, oxidative stress, inflammation, formation of glycation products, and abnormal coagulation $(29,30)$. It is not clear whether these mechanisms influence the progression of atherosclerosis or development of $\mathrm{CV}$ diseases in ESRD patients as in general diabetic patients. There are few studies indicating the adverse effects of hyperglycemia as an atherogenic factor in diabetic ESRD patients. Abnormal lipoprotein metabolism deteriorates in the presence of hyperglycemia and may influence atherosclerosis in diabetic ESRD patients (31). Studies are needed to clarify the atherogenic factors related to hyperglycemia in these patients.

Our results showed an association of hyperglycemia, reflected by higher GA levels, with development of CV diseases, but not with CV mortality. Hyperglycemia may have different effects on the development of CV disease and fatality. Risk factors for $\mathrm{CV}$ disease have two different characteristics: those related to $\mathrm{CV}$ events and those affecting fatality (32). To clarify this suggestion, further studies concerning an association between glycemic control and CV disease are needed.

One previous study showed a significant improvement of outcomes by intensive diabetes education and care management including glycemic control in diabetic dialysis patients (33). Multifactorial intervention aimed at many risk factors including hyperglycemia has been shown to reduce CV risk and microvascular events (34). Intervention of glycemic control alone may be insufficient and intervention for many other risk factors may be required for diabetic ESRD patients.

It has not been established that HbAlc is a reliable marker of hyperglycemia in ESRD patients $(5,13)$. Some previous studies showed a weak correlation between HbA1c levels and mean blood glucose levels in diabetic ESRD patients $(11,13,15)$. HbA1c levels in ESRD patients do not always reflect the same blood glucose levels as those in general diabetic patients because of shortened erythrocyte lifespan, erythropoietin therapy or other factors $(12,13)$. Unreliability of HbA1c levels as a glycemic marker may have some influence on the results concerning associations between $\mathrm{HbA} 1 \mathrm{c}$ levels and prognosis. Careful interpretation is therefore required for studies using HbAlc levels as a marker of glycemic control.

Previous studies have examined HbA1c levels as the sole marker of glycemic control, and some showed an association between poor survival and high HbA1c levels $[\geqq 8 \%$ (9), $\geqq 9 \%$ (35), $\geqq 10 \%$ (6), 12 14\% (8)]. These values indicate extremely poor glycemic control because HbA1c levels tend to be lower in ESRD patients than in general diabetic patients. These results suggest that at least we should avoid such poor control.

The present study included few patients with poor glycemic control of HbA1c more than $8.0 \%$. In contrast to previous studies, glycemic control was relatively good in our patients. In the study by Oomichi et al, mean HbAlc was $7.5 \%$ and patients with HbA1c of more than $8.0 \%$ consisted of $36 \%$ of all patients (9). These differences of glycemic control between the studies might have an influence on the different results concerning associations between HbA1c levels and prognosis.

The other marker of hyperglycemia, GA, is not influenced by erythrocyte lifespan or erythropoietin therapy $(13,14)$. 
One previous study showed that GA levels might reflect blood glucose levels more correctly than HbAlc levels in ESRD patients (15). However changes in albumin turnover influence GA levels. Our patients had dialysis for a period of 12 months or longer and the influence of urinary protein excretion on albumin turnover may be small. Hypoalbuminemia induced by inflammation or malnutrition might influence GA levels in a small number of patients.

The correlation between HbA1c2 and GA2 was significant but weak. This indicates that factors other than blood glucose levels may influence on the levels of both markers. We suggest that the influence of the factors affecting HbA1c levels, such as shortened erythrocyte lifespan or erythropoietin therapy may be greater than those affecting GA levels. HbA1c reflects glycemic control during the preceding 12 months and GA reflects those during the preceding 2-4 weeks (36). These differences may influence the correlations between both markers.

The correlation between HbA1c2, GA2 and blood glucose levels were also significant but weak. The coefficients of correlation ( $r$ ) were almost similar between HbA1c2 and blood glucose levels and between GA2 and blood glucose levels. Our results indicated no superiority of GA for reliability of blood glucose levels. However, we obtained only 3 samples of non-fasting blood glucose levels. More measurements of blood glucose levels with uniform condition may be needed to clarify the correlation between these markers and blood glucose levels.

Unfortunately we could obtain GA on only one occasion. GA reflects short-term glycemic control compared with
HbA1c levels. It is possible that GA levels change to some extent during the period of data collection. Therefore, more measurements of GA levels during a longer duration may be needed to clarify an association between GA levels and prognosis.

There are several limitations to the present study. First, the numbers of the patients were small. Secondly, the variables of glycemic control in this study do not always mean cumulative exposure of hyperglycemia. Long-term follow-up of the markers may be needed to examine the influence of hyperglycemic exposure. Third, other data concerning risk factors for CV complications, including smoking, dyslipidemia and inflammatory markers were not available in this study. However the main variables strongly affecting prognosis were included in this analysis.

In conclusion, markers of hyperglycemia at initiation of dialysis or on chronic dialysis did not predict mortality in diabetic ESRD patients. On the other hand, higher GA levels had an association with development of CV disease. Further studies are needed to clarify the beneficial effect of glycemic control and to establish more reliable methods for assessment of glycemic control in these patients.

The authors wish to thank Toshiaki Suzuki, MD, Toshimasa Takahashi, MD, Kano Narita, MD, and the 14 outpatient dialysis centers for accumulating data for this study. The authors are also indebted to Prof. J. Patrick Barron of the International Medical Communications Center of Tokyo Medical University for his review of this manuscript.

\section{References}

1. The Diabetes Control and Complications Trial Research Group. The effect of intensive treatment of diabetes on the development and progression of long-term complications in insulin-dependent diabetes mellitus. N Engl J Med 329: 977-986, 1993.

2. Ohkubo Y, Kishikawa H, Araki E, et al. Intensive insulin therapy prevents the progression of diabetic microvascular complications in Japanese patients with non-insulin-dependent diabetes mellitus:a randomized prospective 6-year study. Diabetes Res Clin Pract 28: 103-117, 1995.

3. Stratton IM, Adler AI, Neil HAW, et al. Association of glycaemia with macrovascular and microvascular complications of type 2 diabetes (UKPDS 35): prospective observational study. BMJ 321: 405-412, 2000.

4. Nathan DM, Cleary PA, Backlund JY, et al. Intensive diabetes treatment and cardiovascular disease in patients with type 1 diabetes. N Engl J Med 353: 2643-2653, 2005.

5. Feldt-Rasmussen B. Is there a need to optimize glycemic control in hemodialyzed diabetic patients? Kidney Int 70: 1392-1394, 2006.

6. Wu MS, Yu CC, Yang CW, et al. Poor pre-dialysis glycaemic control is a predictor of mortality in typeII diabetic patients on maintenance haemodialysis. Nephrol Dial Transplant 12: 2105-2110, 1997.

7. Morioka T, Emoto M, Tabata T, et al. Glycemic control is a predictor of survival for diabetic patients on hemodialysis. Diabetes Care 24: 909-913, 2001.

8. Tzamaloukas AH, Murata GH, Zager PG, Eisenberg B, Avasthi
PS. The relationship between glycemic control and morbidity and mortality for diabetics on dialysis. ASAIO J 39: 880-885, 1993.

9. Oomichi T, Emoto M, Tabata T, et al. Impact of glycemic control on survival of diabetic patients on chronic regular hemodialysis:a 7-year observational study. Diabetes Care 29: 1496-1500, 2006.

10. Okada T, Nakao T, Matsumoto $H$, et al. Predialysis factors related to prognosis in type 2 diabetic patients on chronicdialysis in Japan. Nephrology 7: 250-256, 2002.

11. Williams ME, Lacson E Jr, Teng M, Ofsthun N, Lazarus JM. Hemodialyzed type I and type II diabetic patients in the US:Characteristics, glycemic control, and survival. Kidney Int 70: 15031509, 2006.

12. Nakao $T$, Matsumoto $H$, Okada $T$, et al. Influence of Erythropoietin treatment on hemoglobin A1c levels in patients with chronic renal failure on hemodialysis. Intern Med 37: 826-830, 1998.

13. Ansari A, Thomas S, Goldsmith D. Assessing glycemic control in patients with diabetes and end-stage renal failure. Am J Kidney Dis 41: 523-531, 2003.

14. Shima K, Ito N, Abe F, et al. High-performance liquid chromatographic assay of serum glycated albumin. Diabetologia 31: 627631, 1988.

15. Chujo K, Shima K, Tada H, Oohashi T, Minakuchi J, Kawashima $\mathrm{S}$. Indicators for blood glucose control in diabetics with end-stage chronic renal disease: GHb vs. glycated albumin (GA). J Med Invest 53: 223-228, 2006.

16. Kouzuma T, Usami T, Yamakoshi M, Takahashi M, Imamura S. 
An enzymatic method for the measurement of glycated albumin in biological samples. Clin Chim Acta 324: 61-71, 2002.

17. Glucose tolerance, and mortality. comparison of WHO and American Diabetes Association diagnostic criteria. The DECODE study group. European Diabetes Epidemiology Group. Diabetes epidemiology:Collaborative analysis of diagnostic criteria in Europe. Lancet 354: 617-621, 1999.

18. Khaw KT, Wareham N, Bingham S, Luben R, Welch A, Day N Association of hemoglobin A1c with cardiovascular disease and mortality in adults:the European prospective investigation into cancer in Norfolk. Ann Intern Med 141: 413-420, 2004.

19. Selvin E, Coresh J, Golden SH, Brancati FL, Folsom AR, Steffes MW. Glycemic control and coronary heart disease risk in persons with and without diabetes:the atherosclerosis risk in communities study. Arch Intern Med 165: 1910-1916, 2005.

20. Shoji T, Kawagishi T, Emoto M, et al. Additive impacts of diabetes and renal failure on carotid atherosclerosis. Atherosclerosis 153: 257-258, 2000.

21. Ishimura E, Shoji T, Emoto M, et al. Renal insufficiency accelerates atherosclerosis in patients with type 2 diabetes mellitus. Am J Kidney Dis 38 (suppl 1): S186-S190, 2001.

22. Sarnak MJ. Cardiovascular complications in chronic kidney disease. Am J Kidney Dis 41 (suppl 5): S11-S17, 2003.

23. Shoji T, Emoto M, Shinohara K, et al. Diabetes mellitus, aortic stiffness, and cardiovascular mortality in end-stage renal disease. J Am Soc Nephrol 12: 2117-2124, 2001.

24. Nishizawa $Y$, Shoji $T$, Maekawa K, et al. Intima-media thickness of carotid artery predicts cardiovascular mortality in hemodialysis patients. Am J Kidney Dis 41 (suppl 1): S76-S79, 2003.

25. Ono K, Tsuchida A, Kawai H, et al. Ankle-brachial blood pressure index predicts all-cause and cardiovascular mortality in hemodialysis patients. J Am Soc Nephrol 14: 1591-1598, 2003.
26. Turina M, Fry DE, Polk HC Jr. Acute hyperglycemia and the innate immune system:clinical, cellular, and molecular aspects. Crit Care Med 33: 1624-1633, 2005.

27. Coughlin SS, Calle EE, Teras LR, Petrelli J, Thun MJ. Diabetes mellitus as a predictor of cancer mortality in a large cohort of US adults. Am J Epidemiol 159: 1160-1167, 2004.

28. Japanese Society for Dialysis Therapy. An overview of regular dialysis treatment in Japan (as of December 31, 2004). Japanese Society for Dialysis Therapy, Tokyo, 2005 (in Japanese).

29. Beckman JA, Creager MA, Libby P. Diabetes and atherosclerosis. Epidemiology, pathophysiology, and management. JAMA 287: 2570-2581, 2002.

30. Ceriello A. Postprandial hyperglycemia and diabetic complications. Is it time to treat? Diabetes 54: 1-7, 2005.

31. Kimoto E, Shoji T, Emoto M, et al. Effect of diabetes on uremic dyslipidemia. J Atheroscler Thromb 9: 305-303, 2002.

32. Nishizawa Y, Shoji T, Ishijima E, Inaba M, Morii H. Paradox of risk factors for cardiovascular mortality in uremia:Is a high cholesterol level better for atherosclerosis in uremia? Am J Kidney Dis 38 (suppl 1): S4-S7, 2001.

33. McMurray SD, Johnson G, Davis S, McDougall K. Diabetes education and care management significantly improve patient outcomes in the dialysis unit. Am J Kidney Dis 40: 566-575, 2002.

34. Gaede P, Vedel P, Larsen N, Jensen GV, Parving HH, Pedersen O. Multifactorial intervention and cardiovascular disease in patients with type 2 diabetes. N Engl J Med 348: 383-393, 2003.

35. Japanese Society for Dialysis Therapy. An overview of regular dialysis treatment in Japan (as of December 31, 2001). Japanese Society for Dialysis Therapy, Tokyo, 2002 (in Japanese).

36. Tahara Y, Shima K. Kinetics of HbAlc, glycated albumin, and fructosamine and analysis of their weight functions against preceding plasma glucose level. Diabetes Care 18: 440-447, 1995.

(C) 2007 The Japanese Society of Internal Medicine http://www.naika.or.jp/imindex.html 